\title{
Guest editorial: ISPC 2015 special issue
}

\author{
Sebastian Fortin ${ }^{1}$. Camilo Martinez Gonzalez ${ }^{1}$. Alfio Zambon ${ }^{2}$. \\ Waldmir Araujo Neto $^{3}$
}

Published online: 11 March 2019

(c) Springer Nature B.V. 2019

The ISPC 2015 symposium was held from July 28th to July 30th 2015 at the Federal University of Rio de Janeiro, Brazil. It gathered experts in philosophy and history of chemistry from different countries around the world, who shared their research results and discussed the current topics of the discipline. This is a volume of "Selected Contributions" on the occasion of that conference. The call for contributions was open not only to those who actually attended the Conference, but also to those researchers that initially planned to attend but were unable to do so. We want to stress that this is not intended to be a volume of proceedings, but a sort of special issue on philosophy and history of chemistry inspired by the talks and discussions given at ISPC 2015. The following articles were selected to include the volume:

The author of the first paper is Marina Banchetti, from the Florida Atlantic University, a regular participant of the ISPC meetings. Banchetti argues that the concern over microstructure, specific of contemporary chemistry, can also be found in the chemical philosophy of Robert Boyle. In particular, she explains how, in spite of his mechanistic philosophy, Boyle considered that the specific microstructure of what he called 'chemical atoms', understood in geometric terms, accounted for the essential properties of chemical substances.

Then, the editor-in-chief of this journal, Eric Scerri deals with five relevant issues in chemical education. He criticizes five ideas that are commonly reported in chemistry textbooks by arguing that they are either wrong or the source of misconceptions: the idea that having $\mathrm{pH} 7$ is a sufficient condition for a solution to be neutral; the use of Le Chatelier principle for making predictions; the order of occupation of $4 s$ and $3 d$ orbitals in transition metals; the claimed stability of half-filled electronic sub-shells; and the placement of La and Ac in the third group of the Periodic Table.

The next paper is by the Argentine researcher Hernán Lucas Accorinti, who analyzes the different definitions of electronegativity as manifesting a case of incompatible models in chemistry. Accorinti proposes a new perspective that incorporates the role of experimental techniques in the very notion of representation; this perspective allows us to understand

Sebastian Fortin

sfortin@conicet.gov.ar

1 CONICET-Universidad de Buenos Aires, Buenos Aires, Argentina

2 Universidad Nacional de la Patagonia San Juan Bosco, Comodoro Rivadavia, Argentina

3 Universidade Federal do Rio de Janeiro, Rio de Janeiro, Brazil 
how supposedly incompatible models of the same target system can both be simultaneously representative.

The fourth paper is from two new contributors to the philosophy of chemistry community, María Silvia Polzella and Penélope Lodeyro, from Argentina, who consider the important topic of computer simulations in quantum chemistry. In particular, they analyze the distinction between ab initio and semi-empirical methods from a philosophical point of view. On this basis, they re-evaluate the characterization of the semi-empirical simulations in this area.

The Colombian philosopher of science Juan Camilo Martínez González is the author of the fifth article, which deals with Hund's paradox, that is, the paradox derived from the fact that quantum mechanics cannot account for the difference between enantiomers of a same substance. The main purpose of this article is to argue for the need to adopt a clear interpretation of quantum mechanics in order to solve such a paradox.

In the sixth paper the Brazilian historian of science Roberto de Andrade Martins focuses his attention on the status of conservation principles in natural sciences, in particular, the principle of the conservation of mass through chemical reactions. The paper reviews Meyerson's views in the historical context of the late 19th and early 20th century, when the principle of mass conservation was tested in highly accurate experiments.

Finally, Jesus Alberto Jaimes Arriaga, Sebastian Fortin and Olimpia Lombardi, from Argentina, discuss the supposed reduction of chemistry to physics in the light of the Quantum Theory of Atoms in Molecules (QTAIM), proposed by Richard Bader. After reviewing the positive and negative aspects of the QTAIM, they argue that it fails to provide the desired reduction of chemistry to standard quantum mechanics. On this basis, the authors suggest that the Bohmian formulation of QM (and its associated ontology) might support the reductionist aspirations of the defenders of the QTAIM.

Publisher's Note Springer Nature remains neutral with regard to jurisdictional claims in published maps and institutional affiliations. 